\title{
An Observation on Curative Effect of Dahuang-Mudan Decoction among Patients Receiving Postoperative Chemotherapy of Colon Carcinoma
}

\author{
Wuxuan Liu $^{1}$ \\ ${ }^{1}$ College of traditional Chinese medicine, Hainan Medical College, Haikou, 571100, China
}

\begin{abstract}
PURPOSE This thesis focuses on the research of the observation of curative effect of Dahuang-Mudan decoction(DMD) applied among the patients receiving chemotherapy in post operation of colon carcinoma. METHOD The selected cases of research have been 130 postoperative patients of colon carcinoma who had acquired hospitalization in some tertiary-grade A class hospital from June 2018 to December 2019, all of which are devided into two respetive groups by random number-- the Routine Group(RG) and the Contrast Group(CG), each containing 65 cases. Patients in RG get solely chemotherapy for rehabilitation, while the use of DMD is being added to patients in CG on the basis of standards in RG. After a certain period of treatment, some contrasts would be done in overall efficacy rate, the first anal exsufflation in post-operation, possibilities of complication occurence and quality evaluation on life. RESULT After related treatment of DMD, patients in CG perform remarkably better in aspects of overall efficacy, the first anal exsufflation in post-operation, possibilities of complication occurence and quality evaluation on life than those in RG. CONCLUSION The use of DMD for postoperative patients of colon carcinoma who are receiving chemical treatment produces remarkable curative effect and vast value of clinical application, thus further promotion and practice could be positively adopted.

Colon carcinoma is clinically a malignant tumor frequently affecting in digestive system, occurring at the connection of sigmoid colon and rectum. With the continueous change of people's diet constructure and tempo of life, the morbidity toll of colon carcinoma has seen a tendency of increase year by year. The age section of most of patients mainly lays between 35 to 55 years old in clinical, while male patients have suffered a higher morbidity than female ones, and the gender ratio could be a near 2:1[1]. The aetiological agent of colon carsinoma could be tightly connected to a diet that is high in fat and low in dietry fiber. A preference for high-fat food with low dietry fiber may incur chronic colonitis and intestinal polyps, which may further deteriorate into colon carcinoma. For clinical front line, the main method for the treatment of colon carcinoma is related operation at its core, along with a variety of postoperative adjuvant therapies. Western medicine tends to adopt chemotherapy, but the clinical effect could be less ideal for the patients while a number of side-effects may also emerge easily. The chinese traditional medicine tends to apply a dialectic therapy at core for the treatment, and the combination therapy including dialectic therapy and chemotherapy has gradually become a mainstream method for the treatment of colon carcinoma due to facual effectiveness of ameliorating curative-effects and reducing side-effects[2]. This research focuses on an analysis of the actual clinical curative-effects of DMD applied on patients who are receiving postoperative chemotherapy of colon carcinoma.
\end{abstract}

\section{RESEARCH DATA AND METHODOLOGY}

\subsection{Clinical Cases}

The selected cases of research have been 130 postoperative patients of colon carcinoma who had acquired hospitalization in some tertiary-grade A class hospital from June 2018 to December 2019, all of which meet the diagnosis criteria of latest "Guidelines for Diagnosis and Treatment of Colorectal Cancer (Version
2018)"[3] and are devided into two respetive groups---RG and $\mathrm{CG}$ by random number, each containing 65 cases. There are 43 male patients and 22 female patients ranging from 33 to 72 years old in RG, on an average of $(49.43 \pm 8.28)$. The duration of morbidity ranges from 0.4 to 3.5 years, on an average of (1.02 \pm 0.32$)$. And the pathological regions of colon differ, with right-sided cases--35 and left-sided ones--30. For $\mathrm{CG}$, there are 40 male patients and 25 female patients ranging from 30 to 75 years old, on an average of (49.43 \pm 8.28$)$, and the duration of morbidity ranges from 0.3 to 3.8 years, on an average of $(1.36 \pm 0.35)$ years. And 
it's with 38 right-sided cases and 27 left-sided ones in it. Making comparisons of the two groups' general clinical data in gender composition, age distribution, morbidity duration, cancerous parts and etc, we could get the result of $\mathrm{P}>0.05$, hence the statistics difference leads to meaninglessness without comparability in statistics.

\subsection{Criteria for Selection and Exclusion}

All the vital signs of selected patients invovled in research are stable with no discoveries of other intensive complications or diseases. Under the guarantee of right to be informed, all the involved patients voluntarily participate in this research and process of rehabilitation to be devided into certain groups. Criteria for selection:(1)With colonoscopy, all the patients had been diagnosed with colon carcinoma. (2)All the patients had been treated with a surgical operation to get tumor excision. (3) Patients Diagnosed as "thermotoxin amassed in bowels" syndrome accroding to dialectic classification in Chinese Traditional Medicine. (4) Patients needing combined chemotherapy in post-operation for rehabilitation. Criteria for exclusion: (1)With colonoscopy, the diagnosis result points to benign colon tumor. (2) Patients with observed metastasis of colon carcinima cells. (3)Patients with severe functional consciousness disorders, or with intensive cardiac, hepatic, pulmonary, renal co-morbidity and complications. (4) Patients with psychological diseases or mental disorders.

\subsection{Therapeutic Method}

Periodic programme of chemotherapy of " $\mathrm{m}$ FALFOX7'[4] would be applied among patients in RG. And an intravenous drip would be processed with $90 \mathrm{mg}$ oxaliplatin and $450 \mathrm{mg}$ calcium folinate for a respective $1.5 \mathrm{~h}$ at the first day of trial. And then another $500 \mathrm{mg}$ fluorouracil would be pumped intravenously for a totality of another $48 \mathrm{~h}$. A single treatment cycle would include 30 days and during the whole rehabilitation cycle there would be at least more than twice of chemotherapy. Application of DMD would be added to CG patients under the basis of the RG patients, a copy of formula of DMD contains 20g Fructus Aurantil, 20g Sculellaris Barbata, 20g Codonopsis Pilosula, 10g Semen Persicae, 25g Moutan Cortex, 15g Semen Benincasae, 68g Radixet Rhizoma Rhei Vinata, 25g Magnolia Officinalis, $15 \mathrm{~g}$ Wolfiporia Cocos, 10g Platycodon Grandiflorus, $15 \mathrm{~g}$ Atractylodis Macrocephalae Rhizoma, 20g Hydiotis Diffusa Willd, 20g Natrii Sulfas and 25g Semen Coicis Crudum. The herbs are provided by the hospital's CTM department and the process of decoction has been managedand supervised by it too. Every patient will take the DMD for $200 \mathrm{ml}$ per time for twice a day with a treatment cycle of 30 days.

\subsection{Clinical Indicators for Observation}

Observation and comparison on the two groups would be made in overall efficacy rate, the first anal exsufflation in post-operation, possibilities of complication occurence, and a scoring criteria of functional status will also be set in use of Karnafsky (KPS) to evaluate the life quality of involved patients.

\subsection{Criteria for Curative-effect Evaluation}

According to the Response Evaluation Criteria in Solid Tumors(RECIST)[6] released by the World Health Organization(WTO), evaluation of curative-effect of the patients would be made after 30 days' treatment by a CT scan of abdomen and a test of MRI in radiography to assess the load of solid tumor. The followings are criteria: Patients with remarkable improvements(PWRI): All the ill-health symptoms have disappeared with a decrease of $70 \%$ internal tumor load at least; Patients with improvements(PWI): Symptoms and signs have been largely ameliorated with a decrease of $50 \%$ internal tumor load at least. Patients with SD(PWSD): No other noticeable symptoms or signs have been observed with a decrease of $50 \%$ tumor load or an increase of $25 \%$ tumor load at most. Patients with aggravation(PWA): Symptoms and signs have been tangibly deteriorated compared with pre-treatment status with an increase of $25 \%$ tumor load at least or an appearance of novel nidus. Overall efficacy rate $(\%)=($ PWRI + PWI + PWSD $) /$ Total quantity $\times 100 \%$

\subsection{Statistical Analysis}

All the acquired information of clinical data will be analyzed with SPSS 18.0(a statistical-analysis software). The research uses "s" to represent "measurement data" with test of " $t$ ", and the unit "rate" used to represent "enumeration data" with test and analysis of " $\chi 2$ ". " $P$ " will be used to represent statistical significance, when $\mathrm{P}<0.05$, it's with significant statistical difference and thus comparability of statistical analysis would be made.

\section{RESULTS}

\subsection{Comparisons to Overall Clinical Efficacy Rate of Patients}

Overall efficacy rate(OER) of $\mathrm{CG}$ has been tangibly higher than it in $R G, P<0.05$, it's with significant statistical difference and thus comparability of statistical analysis could be made. Details would be listed in following chart.

\begin{tabular}{lllllll}
\multicolumn{6}{c}{ Chart 1 Comparison of OER in RG and CG after 30 days' treatment } \\
\hline GROUP & CASES & PWRI & PWI & PWSD & PWA & OER (\%) \\
& & & & & & \\
\hline RG & 65 & 4 & 18 & 25 & 18 & 72.31 \\
CG & 65 & 19 & 27 & 16 & 3 & 95.38 \\
\hline
\end{tabular}




\subsection{Comparison to The First Anal Exsufflation of Postoperative Patients}

The average time point of the first anal exsufflation among patients in $\mathrm{CG}$ is $(50.61 \pm 7.36) \mathrm{h}$, significantly lower than that in RG of $(79.43 \pm 11.78) h$. It's with significant statistical difference and thus comparability of statistical analysis could be $\operatorname{made}(\mathrm{t}=7.739$, $\mathrm{P}=0.001<0.05)$

\subsection{Comparison to The Possibilities of Complication Occurance During Treatment}

During rehabilitation, the rate of complication occurance among patients in CG is $4.64 \%$ (3 cases), significantly lower than that in RG of $24.61 \%$ (16 cases). It's with significant statistical difference and thus comparability of statistical analysis could be $\operatorname{made}(\chi 2=5.378$, $\mathrm{P}=0.016<0.05$ )

\subsection{Comparison to Evaluation of Life Quality}

Before rehabilitation, comparison of the two groups had been made through KPS, resulting in insignificance of statistical difference $(\mathrm{P}>0.05)$; After 30 days' rehabilitation, KPS in CG is tangibly higher than that in RG, It's with significant statistical difference and thus comparability of statistical analysis could be made $(\mathrm{P}<0.05)$. Details would be listed in following chart.

\begin{tabular}{llllll}
\multicolumn{5}{c}{ Chart 2 } & The Comparison to KPS in Both Pre-and-post \\
\hline GROUP & CASES & PRE- & POST- & t-value & P-value \\
\hline RG & 65 & $68.74 \pm 8.87$ & $71.47 \pm 8.47$ & 4.703 & 0.000 \\
CG & 65 & $68.97 \pm 8.54$ & $79.74 \pm 7.63$ & -3.956 & 0.001 \\
t-value & & -0.328 & -9.437 & & \\
P-value & & 0.843 & 0.001 & & \\
\hline
\end{tabular}

\section{DISCUSSION}

A method of combined treatment of both operation and chemotherapy is usually applied clinically to treat with malignant tumor in recent practice, however, severe lesion which is irreversible may be caused to normal cells of certain organism when eliminating cells of malignant tumor. The most common chemotherapy method for clinical treatment of patients with colon carcinoma is to combine flourouracil, oxaliplatin and calcium folinate. The antitumor mechanism of oxaliplatin(a new platium-based antitumor drug) is that it will act upon the bimolecular chain of DNA via the binding interaction of hydrated derivatives, thus forming an interaction between intra-chain and inter-chain to inhibit the DNA synthesis of tumor cells and then to develop activity in the process of anti-tumor-cells[7]. Flourouracil works effectively in clinical among all sorts of cancers in digestive system, and the antitumor mechanism for it is to inhibit the activity of thymidylate synthase to further inhibit the DNA synthesis of tumor cells, what's more, there would also be certain interference to the synthesis process of RNA caused by flourouracil[8]. The combination use of flourouracil and calcium folinate will synergize and effectively reduce maglinant tumor cells[9].

The chemotherapeutic effect of Western Medicine mainly locates in "exhortation", while CTM aims to ameliorate the curative effect and reduce side-effect. Thus the improvisional therapy combining Western Medicine and CTM emerges as the time requires. This research focuses on the clinical use of DMD for patients' rehabilitation, Dahuang(Radixet Rhizoma Rhei)could help clear inner heat and detoxify, while Mudan bark(Moutan Cortex) could help clear inner heat as well as dissipate stasis. The compatibility of these two herbs could intensify the above effect. Natrii Sulfas could help digest, remove retained water and milden purge; Semen Persicae could help invigorate the circulation of blood and moisten the digestive apparatus; Semen Benincasae functions in curing appendititis, could help promote the circulation of $\mathrm{Qi}$, moisten the digestive apparatus and drain the pus out; The compatibility of Fructus Aurantil and Magnolia Officinalis could help circulate Qi in Xiajiao(the part of the body cavity below the umbilicus, housing the bladder, kidneys and bowels), and help invigorate the circulation of blood; Wolfiporia Cocos could help tonify spleen and remove dampness; Atractylodis Macrocephalae Rhizoma could help tonify Qi and spleen; Codonopsis Pilosula could help tonify Qi and strengthen the body resistance; Sculellaris Barbata and Hydiotis Diffusa Willd could help clear heat and detoxify; Semen Coicis Crudum could help tonify the spleen and drain the pus out; Platycodon Grandiflorus is targeting for the lung meridian while the lung is interlinked with large intestine, it can direct the components into the meridian while draining the pus out and eliminating phlegm. The compatibility of the above herbs could help clear inner heat, detoxify and cure appendititis.

The reslut has showed that the overall efficacy rate of $\mathrm{CG}$ in clinical is tangibly higher than that in $\mathrm{RG}$, which means the use of DMD among colon-carcinoma patients receiving postoperative chemotherapy has worked effectively.. One month after treatment, via KPS, the evaluation of patients in CG is tangibly higher than that in $\mathrm{RG}$ as well, signaling DMD progressing the improvement of life quality among patients receiving postoperative chemotherapy. After 30 days' treatment, the first duration of anal exsufflation of patients in CG is tangibly shorter than that in RG, signaling DMD progressing the rehabilitation of patients' function of gastrointestinal tract positively, and it could shorten the duration as well, reflecting the effect of Fructus Aurantil, Magnolia Officinalis and Natrii Sulfas of circulating the 
Qi . During the treatment, the possibility of complication occurance of patients in $\mathrm{CG}$ is also tangibly lower than that in $\mathrm{RG}$, signaling DMD helping ameliorate effectiveness and reduce side-effect.

To sum up, the curative effect of DMD is remarkable among patients receiving postoperative chemotherapy, the CTM could also play a role in cultivating healthy atmosphere in organic body, which will ameliorate effectiveness and reduce side-effect along with chemotherapeutic drugs. The clinical value is high thus could be promoted positively.

\section{REFERENCES}

1. Jing Zhiliang, Xu Yanhua. Clinical Research on Jiedu Kang ' ai Decoction Combined with Cetuximab in Treatment of Advanced Colorectal Cancer and its Influence on Immune Function and Quality of Life[J]. Journal of Hubei University of Chinese Medicine, 2016,18(2) :25-28.

2. Ren Yi. Clinical Observation on Modified Dahuang Mudan Decoction Combined with Western Medicine in the Treatment of Acute Pancreatitis[J]. Journal of Traditional Chinese Medicine,2018 (08) :1178-1180.

3. Expert Group of Guidelines for Diagnosis and Treatment of Colorectal Cancer (Version 2018) of National Health and Family Planning Commission of PRC.Guidelines for Diagnosis and Treatment of Colorectal Cancer (Version 2018) [J].Chinese Journal of Clinicians(Electronic Edition), 2018, 1 (1) :3-23.

4. Zhao Zheng, Ran Feiwu, Zhang Xiaoxue, etc. An Analysis on Curative-effect of Advanced Colon Carcinoma with m FALFOX7[J]. Journal of Modern Oncology, 2015, 18 (7) :1374-1375.

5. He Xiaoning. A Comparison to Criteria of Synthetic Curative-effect of CTM and Response Evaluation Criteria in Solid Tumors(RECIST) with Advanced Colorectal Carcinoma[D].Beijing: Bejing University of Chinese Medicine, 2010.

6. Yang Xuening, Wu Yilon. RECIST-New Guidelines to Evaluate The Response to Treatment in Solid Tumors[J].The Journal of Evidence-Based Medicine, 2014,4(2) :85-90, 111.

7. Fang Xiao, Yi Hua, $\mathrm{Hu}$ Tenghui, etc.Effect of Oxaliplatin on Expression of Drug Resistant Gene in DNA Damage Repair in Human Colon Cancer Cells[J].Journal of Clinical Medicine in Practice, 2017, 21 (1) :1-4.

8. Man Yuan. A Research on The Curative-effect of A Combined Treatment of Oxaliplation And Flourouracil for Colon Carcinoma[J],Health Medicine Research and Practice in Higher Institutions, 2016, 13 (5) :55-56, 59.

9. Chen Guoping, Zhu Xiongwen, Chen Zaiping, etc. A Clinical Comparison to Low-dosage And High-dosage Calcium Folinate among Patients of Metastatic Colon Carcinoma. Chongqing Medical Journal, 2017, 46 (32) :4569-4571.
10. Zheng Yanyi, Wen Ruyan, Luo Xia, etc.In-vitro Effect of Dahuang Mudan Decoction on Intestinal Flora[J]. Journal of Guangzhou University of Traditional Chinese Medicine,2016, 33 (3) :357-361.

11. Wang Wei. 32 Cases of Appendititis Treated with DMD $[J]$.Chinese Medicine Modern Distance Education of China, 2014, 12 (12) :50-51. 